ISSN 2079-9292

www.mdpi.com/journal/electronics

Review

\title{
Multicarrier Spread Spectrum Modulation Schemes and Efficient FFT Algorithms for Cognitive Radio Systems
}

\author{
Mohandass Sundararajan * and Umamaheswari Govindaswamy \\ Electronics and Communication Engineering Department, PSG College of Technology, \\ Peelamedu, Coimbatore-641 004, India; E-Mail: gumabhaskar@yahoo.co.in \\ * Author to whom correspondence should be addressed; E-Mail: mohandass25@yahoo.com; \\ Tel.: +91-422-242-4265; Fax: +91-422-257-3833.
}

Received: 17 May 2014; in revised form: 2 July 2014 / Accepted: 4 July 2014 /

Published: 17 July 2014

\begin{abstract}
Spread spectrum (SS) and multicarrier modulation (MCM) techniques are recognized as potential candidates for the design of underlay and interweave cognitive radio (CR) systems, respectively. Direct Sequence Code Division Multiple Access (DS-CDMA) is a spread spectrum technique generally used in underlay CR systems. Orthogonal Frequency Division Multiplexing (OFDM) is the basic MCM technique, primarily used in interweave CR systems. There are other MCM schemes derived from OFDM technique, like Non-Contiguous OFDM, Spread OFDM, and OFDM-OQAM, which are more suitable for CR systems. Multicarrier Spread Spectrum Modulation (MCSSM) schemes like MC-CDMA, MC-DS-CDMA and SS-MC-CDMA, combine DS-CDMA and OFDM techniques in order to improve the CR system performance and adaptability. This article gives a detailed survey of the various spread spectrum and multicarrier modulation schemes proposed in the literature. Fast Fourier Transform (FFT) plays a vital role in all the multicarrier modulation techniques. The FFT part of the modem can be used for spectrum sensing. The performance of the FFT operator plays a crucial role in the overall performance of the system. Since the cognitive radio is an adaptive system, the FFT operator must also be adaptive for various input/output values, in order to save energy and time taken for execution. This article also includes the various efficient FFT algorithms proposed in the literature, which are suitable for CR systems.
\end{abstract}

Keywords: spread spectrum; multicarrier modulation; underlay cognitive radio; interweave cognitive radio; fast fourier transform 


\section{Introduction}

The spectrum requirements for wireless multimedia applications have increased exponentially, leading to spectrum scarcity. However, recent studies show that most of the licensed spectrum is underutilized. Cognitive radio (CR) has been proposed to bridge the gap between spectrum scarcity and underutilization of the available spectrum [1]. The concept of cognitive radio enables coexistence of the legacy systems and new users, which are called primary users (PU) and secondary users (SU) through dynamic spectrum access [2,3]. $\mathrm{CR}$ is an intelligent radio that has two important characteristics namely, observation and adaptability. The observation characteristic means that the CR can observe and learn from the radio environment through spectrum sensing, channel estimation, and modulation type identification. The adaptability characteristic shows that the CR is able to change its communication protocols and parameters through channel allocation, power allocation, modulation and coding scheme selection, and waveform adaptation, based on its observation results [4]. There are three major types of cognitive radio systems: interweave, underlay and overlay CRs. In the interweave type cognitive radio systems, the secondary devices detect the presence of primary user's signal through spectrum sensing [5] and transmit opportunistically only when there is no primary user transmission. In the underlay cognitive radio systems, secondary users are allowed to communicate along with primary users, as long as the interference created to the primary system is below some predefined threshold. The secondary users detect the presence of primary users and control their transmission power accordingly, in the underlay CR systems. Beamforming, MIMO and spread spectrum (SS) techniques are generally used to control the interference to primary users. Finally, in the overlay systems the secondary users transmit concurrently with the primary users. The key enable of the overlay system is that, the secondary user can split its power for secondary communication part and the remainder of the power to assist primary transmissions by relay operation. The power split is done in such a way that, the increase in a primary user's signal to noise ratio (SNR) due to the relay operation of the secondary user, is exactly offset by the decrease in the primary user's SNR due to the interference caused by secondary user's transmit power that is used for secondary communication.

Single carrier modulation, multicarrier modulation and spread spectrum modulation are the most common types of modulation schemes used in wireless communication systems. In single carrier modulation, one radio frequency carrier is used to carry the information. In multicarrier modulation, multiple carriers at different frequencies are used to carry the information, sending a group of bits on each sub-channel. Multicarrier modulation is similar to Frequency Division Multiplexing (FDM) wherein all of the sub-channels are dedicated to a single data source. There are various advantages of multicarrier modulation schemes over single carrier modulation such as bandwidth efficiency and simplicity in the design of equalizers. In wireless channels, multipath propagation of signals leads to fading effects. Fading results in intersymbol interference (ISI), which limits high data rate wireless communications. The multicarrier modulation schemes (MCM) are used to overcome ISI by converting high-rate data stream into multiple low-rate data streams. Thus, MCM techniques are suitable for high data rate CR applications, in multipath fading channel conditions. MCM schemes such as Orthogonal Frequency Division Multiplexing (OFDM) and Filter Bank Multicarrier Modulation (FBMC) have been recognized as potential candidates for the physical layer of interweave cognitive radio systems [6]. Adaptive transceiver architecture based on MCM provides flexibility and 
reconfigurability to cognitive radio systems. In a DSA network [7], the unused frequency bands may be non-contiguously available for the secondary users. Using MCM schemes, the subcarriers coinciding with the portions of the spectrum that are not used by the primary users are assigned to secondary users in order to fill the spectrum holes [8]. In spread spectrum modulation the transmitted signal bandwidth is much larger than the minimum bandwidth required for the signal. Spreading of the signal is accomplished by multiplying the data bits with pseudo-noise sequence or by varying the frequency of transmission according to the pseudo-noise sequence. DS-CDMA is a spread spectrum modulation technique that is commonly used in underlay cognitive radio systems. In DS-CDMA, data rate and power level are controlled based on the spectrum availability and interference constraint on the primary user. In DS-CDMA based underlay cognitive radio systems, the secondary CDMA users spread their data using a signature sequence and the primary users can be CDMA or non-CDMA users. The transmission power and signature sequence of the secondary users are varied adaptively in a dynamic spectrum access (DSA) network. There are various multicarrier spread spectrum modulation (MCSSM) schemes proposed in the literature, such as Multicarrier Code Division Multiple Access (MC-CDMA) and Multicarrier Direct Sequence Code Division Multiple Access (MC-DS-CDMA), which combine the advantages of both MCM and SS schemes. MCSSM schemes are used in both interweave and underlay CR systems. This article gives an exhaustive survey of various SS, MCM and MCSSM schemes.

The two basic elements of cognitive radio systems are spectrum sensing mechanism and adaptive transceiver architecture. Through spectrum sensing mechanism, a secondary user finds the unused spectral holes which can be used for its transmission without causing interference to the primary users. When the nature of the primary user's signal is known, spectrum sensing can be performed based on feature detection and when such knowledge is not available, energy detection is used for spectral analysis. An energy detector can be implemented using Fast Fourier Transform (FFT) by averaging the frequency bins. By increasing the FFT size $N$, the frequency resolution can be improved and it helps in detection of narrowband signals. FFT, as a part of multicarrier modem can be used for spectral analysis [6] in order to identify the spectral holes, without any extra hardware or software. IFFT and FFT are the most computationally intensive blocks in multicarrier modulation schemes. CR systems require efficient FFT/IFFT algorithms in order to save time and energy. In this paper, various efficient FFT/IFFT algorithms proposed in the literature for cognitive radio applications are investigated.

The remainder of this contribution is organized as follows. In Section 2, variants of OFDM based MCM schemes are described. In Section 3, variants of multicarrier CDMA based modulation schemes are described. Section 4 elaborates the efficient FFT algorithms for multicarrier modulation schemes. In Section 5, a summary of efficient FFT algorithms is provided. Section 6 contains the conclusion.

\section{OFDM Based Multicarrier Modulation Schemes}

Weiss et al. [9] proposed OFDM as a promising candidate for a flexible spectrum pooling system. The advantages of using OFDM in cognitive radio systems were discussed in [10]. OFDM reduces the time dispersion effect of multipath channels encountered with high data rates, by using cyclic prefix (CP). A cyclic prefix of length greater than the maximum delay spread of the channel is generally used with OFDM to overcome intersymbol interference. This CP-OFDM modulation transforms a frequency 
selective channel into a group of several flat fading channels, leading to a one tap zero-forcing (ZF) equalization per sub-carrier and thus reduces the requirement for complex equalizers. Advantages of OFDM include high spectral efficiency, robustness against narrowband interference (NBI), easy implementation using FFT and reconfigurability of transmission parameters. These advantages, together with the spectrum sensing capability using FFT and spectrum shaping capabilities, make OFDM the most appropriate transmission technology for CR systems. Allocation of radio resources is a major challenge in OFDM systems. In [11,12], the problem of optimal resource allocation for an OFDM based CR system has been addressed. In [13], the authors have proposed an improved joint subcarrier and bit allocation scheme for cognitive user with primary users' cooperation, in an OFDM based underlay CR network. The authors investigated the performance of cognitive spectral efficiency and the average interference power level. Using numerical analysis it was shown that, the SU's spectral efficiency increases significantly and the interference power level at the PU receiver decreases dramatically for the proposed scheme compared with the traditional non-cooperation scenario.

There are some limitations of OFDM in CR networks [10]. The drawbacks of conventional OFDM systems include large side-lobes of the modulated OFDM subcarriers and large peak-to-average power ratio (PAPR) [6]. The side-lobes result in significant adjacent channel interference among different secondary users and between primary users and secondary users. Large PAPR leads to nonlinear distortion of OFDM signals at the transmitter power amplifier. In [14-19], various sidelobe suppression techniques and PAPR reduction techniques were proposed for OFDM based CR systems.

\subsection{Non-Contiguous OFDM}

Cognitive radios need transceiver designs which use non-contiguous portions of the spectrum for transmission [20]. In OFDM based cognitive radio systems, the transmission bandwidth must be large in order to support high data rates. However, a large contiguous bandwidth may not be available for opportunistic transmission of CR. In addition, in a dynamic spectrum access (DSA) network, multicarrier-based cognitive radio transceivers need to deactivate some of their subcarriers to avoid causing interference to primary users, as shown in Figure 1. To provide high data rates while avoiding interference with incumbent user transmission, a variant of OFDM called non-contiguous OFDM (NC-OFDM) was proposed in [21,22]. The schematic of an NC-OFDM transceiver is shown in Figure 2. Selective subcarrier allocation (SSA) is performed at the transmitter side, where zeroes are assigned for deactivated subcarriers and data symbols are assigned for active subcarriers, as shown in Figure 3. Similarly, selective subcarrier retrieval (SSR) is performed at the receiver side. This modulation method achieves high data rate transmission through collective utilization of non-contiguous blocks of subcarriers and also avoids interference with incumbent users by deactivating subcarriers overlapping with the primary user's signal spectrum. Figure 4 shows the spectrum of a NC-OFDM signal, in which the blue trace is the cognitive radio's environmental signal, the red trace is the OFDM waveform, and the green trace is the narrowband interferer, which can be seen in the middle of the OFDM spectrum. 
Figure 1. Adaptive subcarrier deactivation in the middle of $U$ subcarriers.



Figure 2. Schematic of an NC-OFDM transceiver.

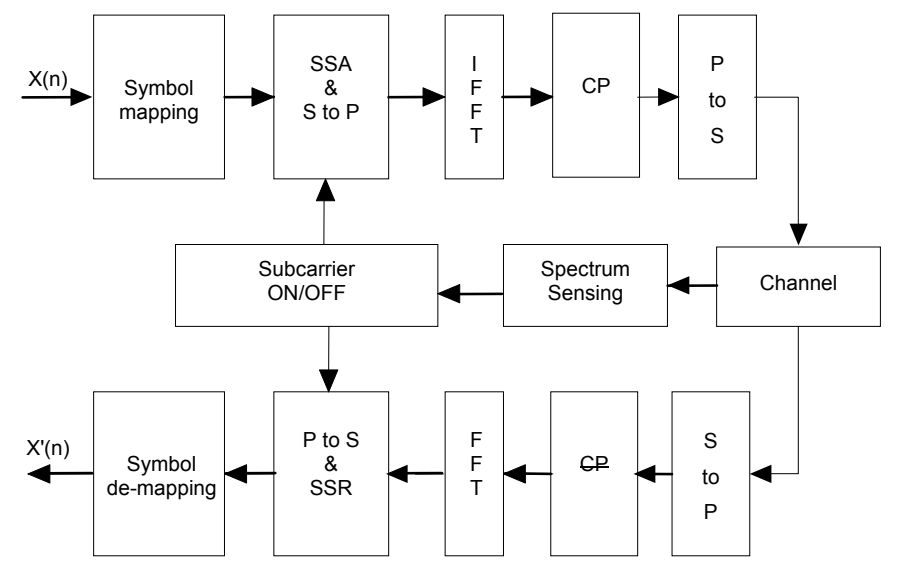

Figure 3. Selective Subcarrier Allocation (SSA) in NC-OFDM.

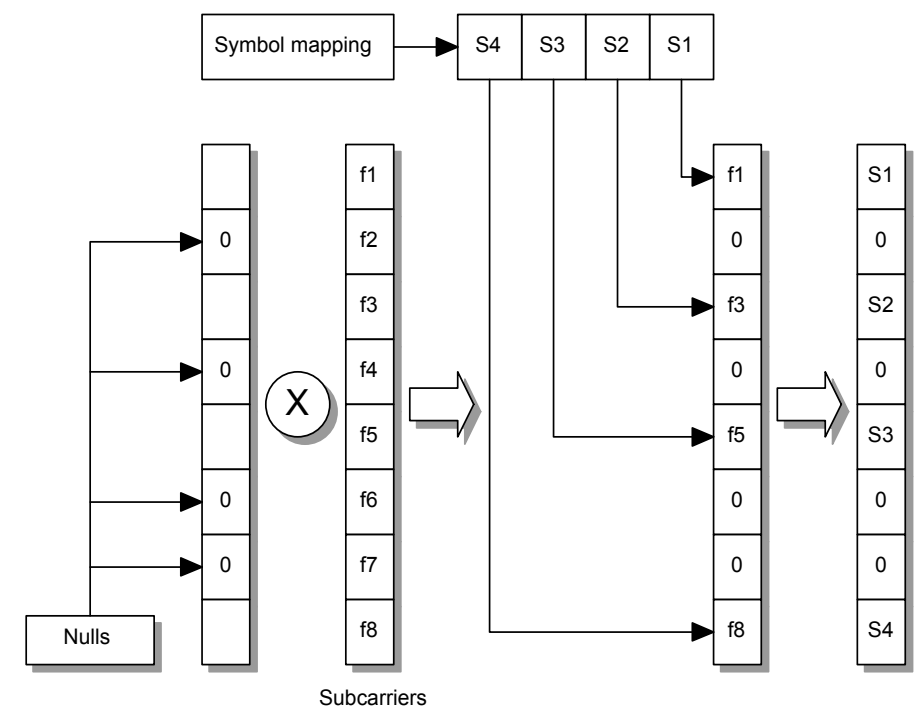


Figure 4. Spectrum of NC-OFDM signal.

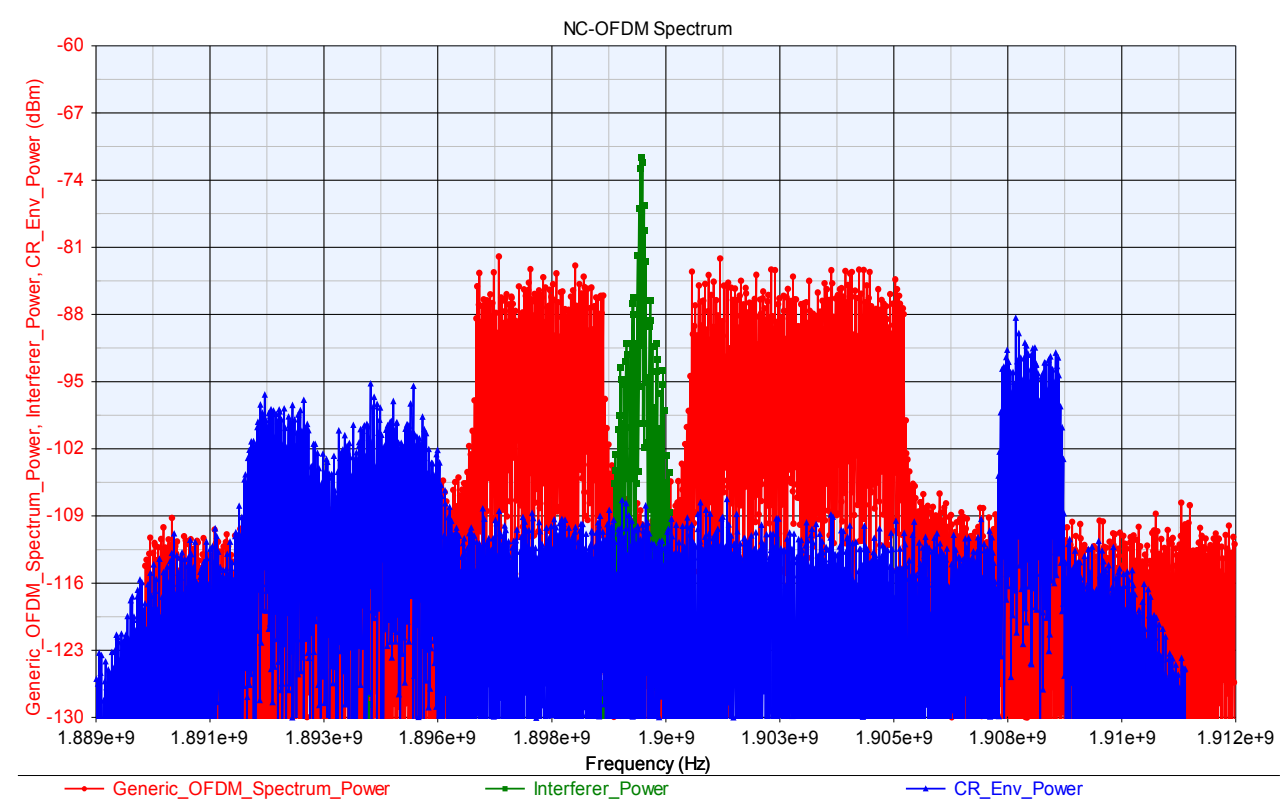

\subsection{Spread OFDM}

Park et al. [23] proposed Walsh-Hadamard Transform-Spread OFDM (WHT-OFDM) scheme to reduce PAPR of conventional OFDM. Properties and advantages of WHT-OFDM method were further studied in [24-26]. In WHT-OFDM, Walsh-Hadamard Transform is added to the OFDM system in order to achieve diversity gain in a mobile environment through spreading of data and hence, it improves the BER performance of the system in multipath fading channels.

Li et al. [27] proposed two novel spread coding schemes for NC-SOFDM for cognitive radio in a DSA network. The proposed spreading code sets eliminate the loss of orthogonality problem in WHT spread OFDM system and maintain the same data rate as that of the traditional OFDM. In one scheme, the whole multicarrier system is divided into multiple subsystems and variable-length orthogonal $\mathrm{WH}$ codes are used for each subsystem to maintain the data rate. In the other scheme, orthogonal carrier interferometry (CI) codes are used for spreading. CI codes are complex spreading codes and they exist for code length of any integer. Thus, Non-contiguous SOFDM (NC-SOFDM) systems with CI spreading code can eliminate the loss of orthogonality among spreading codes caused by deactivating subcarriers and hence CI-SOFDM systems are more suitable for DSA network.

Zhang [28] introduced Non-Continuous CI (NCI) codes into the NC-OFDM system to improve the performance in terms of Bit-Error-Rate (BER) and PAPR reduction. Moreover, the number of users supported in a DSA network can be increased by using Pseudo Carrier Interferometry (PCI) code sets, which have twice the number of codes compared with traditional CI code sets [29]. Discrete Fourier transform-spread OFDM (DFT-S-OFDM) was proposed to be one of the most promising modulation schemes for cognitive radio systems in [30]. Recently, DFT-S-OFDM has been proposed for 3GPP Long Term Evolution (LTE) systems [31]. In DFT-S-OFDM scheme, data symbols are pre-coded using a discrete Fourier transform operation at the transmitter before being sent to the OFDM modulator, in order to reduce PAPR. 


\subsection{Filter Bank Multicarrier Modulation}

Spectral leakage in conventional OFDM due to large side lobes degrades the performance of OFDM-based cognitive radio systems. Therefore, null sub-carriers or guard bands are inserted at the frequency boundaries of OFDM systems in order to avoid adjacent channel interference. Null sub-carriers cause loss of spectral efficiency. Moreover, cyclic prefix in conventional OFDM contains redundant information which adds to loss of spectral efficiency. To overcome these drawbacks, OFDM-offset quadrature modulation (OFDM/OQAM) has been proposed to be a good alternative in [6]. It does not use any $\mathrm{CP}$ and also it offers the possibility to use different prototype filters that eliminate the need for guard bands. Thus, filter bank based OFDM-OQAM maximizes the secondary users' spectral efficiency in a DSA network. Cosine modulated multitone (CMT)-based Cognitive Radio (CMTCR) and filtered multitone (FMT)-based Cognitive Radio (FMT-CR) systems have been studied in [32]. CMT allows overlap of adjacent bands and thus, it maximizes bandwidth efficiency. By limiting the overlaps to adjacent bands, the filter design is simplified. In CMT, PAM symbol sequences are transmitted using vestigial sideband (VSB) modulation. Filtered multitone (FMT) is another multicarrier modulation technique that uses subcarrier bands with no overlap, unlike OFDM-OQAM and CMT which allow for overlapping of adjacent subcarrier bands. Data symbols are quadrature amplitude modulated (QAM). Guard bands are used to separate subcarrier bands, which results in loss in bandwidth efficiency compared to OFDM-OQAM and CMT. Filter bank (FB) based spectrum analyzer has been proposed in [6], as an alternative to FFT based spectrum sensing. It was shown that, the FB spectrum analyzer can provide higher spectral dynamic range for spectrum sensing and hence, it can be used for detecting even low power primary users.

\section{Multicarrier CDMA Based Modulation Schemes}

There are various spectrum sharing approaches proposed in the literatures for CDMA based cognitive radio systems. In [33,34], the authors have proposed an interference-minimizing code assignment strategy for the secondary users in the CDMA underlay cognitive radio networks. The interference caused from the existing primary and secondary users is minimized by minimizing the mean square cross-correlations between the candidate codes and the received signal. The authors demonstrated that the proposed code-assignment strategy exhibits significant performance gains over random code-assignment strategy. In [35], the authors investigated the relationship among the interference threshold settings, the transmission rate of secondary links and the transmission power of the primary links in a CDMA based underlay cognitive wireless network. Given the interference threshold, the maximum achievable rate for the secondary links is found by formulating an optimization problem. In [36], the authors proposed a two-phase channel and power allocation scheme that improves the total number of subscribers that can be simultaneously served by the cognitive radio base stations. The channels and power are allocated to base stations such that the total coverage is maximized while maintaining the interference caused to each primary user below a prescribed limit. The authors of [37] proposed the adaptive spectrum sharing schemes namely intrusive and non-intrusive spectrum sharing, for CDMA based cognitive medium access control (MAC) in the uplink communications over the cognitive radio networks. The secondary users can adaptively switch 
between the schemes based on the channel utilization, traffic load and interference power constraints of primary users. The secondary users are allowed to transmit either in underlay mode or interweave mode based on the availability of the primary users. In [38], the authors proposed an iterative least square based primary user identification algorithm for detecting the signature codes utilized by the primary users in a CDMA network. Based on the availability of code channels, the secondary users are allocated with the channels and power simultaneously. The advantage of the proposed method is that, it does not require any prior knowledge about the primary CDMA network. In [39], the authors investigated the design of channel-aware access control (CAAC) algorithms to protect the primary user transmission, in cognitive radio networks. In their proposed algorithm, the access probabilities of secondary users are adjusted according to the channel-state information and the measured interference temperature. The authors have demonstrated the convergence and effectiveness of the distributed CAAC algorithms, in the work. In [40], the authors investigated the joint power and rate allocation strategies for CDMA based underlay cognitive radio networks with heterogeneous traffic. In their contribution, a combination of streaming and elastic traffic is considered and a cross-layer resource allocation is proposed. Max-min and proportional fairness (PF) approaches are compared for the optimization problem and it was demonstrated that, PF outperforms max-min approach in both homogenous and heterogeneous traffic conditions. In [41], the authors have investigated two joint admission and power control (JAPC) algorithms namely, minimal revenue efficiency removal (JAPC-MRER) and Minimal signal strength removal algorithm (JAPC-MSSRA) in the presence and absence of log-normal shadowing. The authors studied the combined effect of path loss and log-normal shadowing on total secondary revenue and blocking probability and also compared the performance of the two algorithms for shadowing and non-shadowing cases.

In [42], the authors proposed a new cognitive radio network model based on CDMA and evaluated the performances of secondary users and primary users in terms of outage probability, blocking probability, BER and average data rate of secondary users. The authors have also proposed two new spectrum sensing schemes based on interference limit and compared their performance with existing adaptive spectrum sensing scheme. In [43], the authors investigated the performance of CDMA based cognitive radio networks in the presence of receive beamforming at the base stations, for uplink communication. Using the simulation results the authors have shown that, beamforming results in significant performance improvements in terms of probability of outage. In [44], a modified greedy algorithm was proposed to vary the number of activated secondary users based on the pre-determined interference threshold, in a DS-CDMA based cognitive radio network. Using simulation results it was shown that, the proposed CR system exhibits an acceptable BER performance at low SNR level when compared to conventional CDMA system without Greedy algorithm. In this section, various multicarrier CDMA (combination of OFDM and CDMA) based modulation schemes are discussed.

\section{1. $M C-C D M A$}

Sarath et al. [45] proposed Multi Carrier Code Division Multiple Access (MC-CDMA) as a promising technique for cognitive radio systems by exploring the reconfigurability options in MC-CDMA. MC-CDMA system is able to mitigate the ISI and the multiuser interference (MUI) in the frequency selective multipath fading channels by combining OFDM and CDMA techniques. The MC-CDMA 
transmitter spreads the original data stream over different subcarriers in frequency domain, using a spreading code [46]. Multiple secondary users are assigned with orthogonal spreading codes to guarantee their separability at the receiver. The reconfigurability options in MC-CDMA systems include spreading code type and length, FFT size, number of active subcarriers, modulation method, bandwidth, data rate, channel coding, forward error correction and number of simultaneous users. A reconfigurable MC-CDMA transmitter schematic is shown in Figure 5.

Figure 5. Reconfigurable MC-CDMA transmitter.

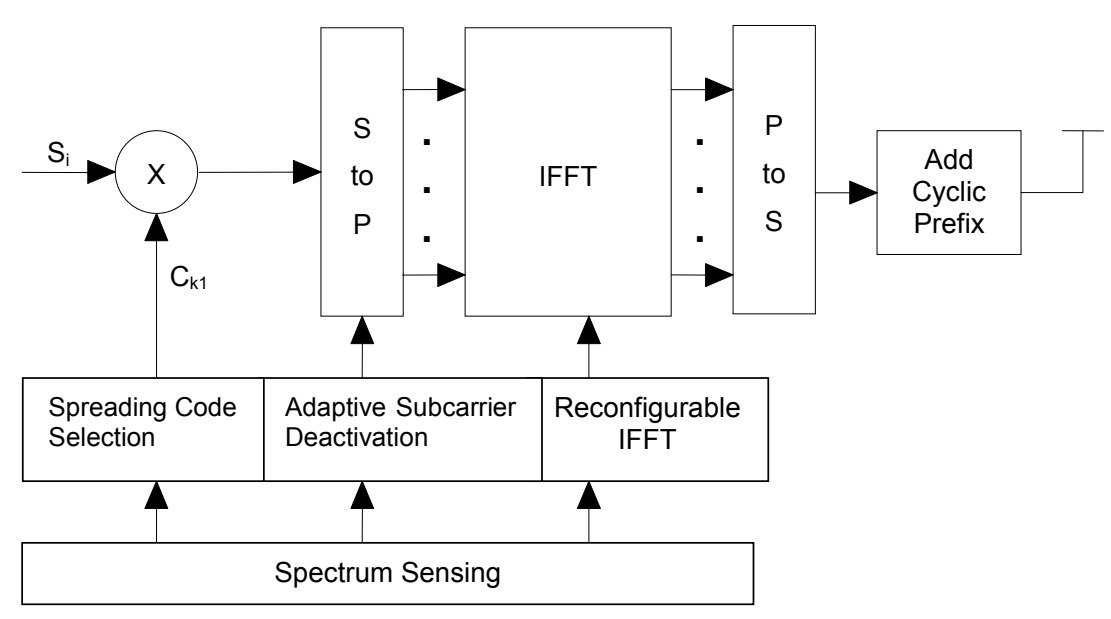

In a CR network, interference between MC-CDMA based secondary users and primary users can be avoided by using the subcarrier deactivation technique, similar to Non-Contiguous OFDM (NC-OFDM) and such schemes are called Non-Contiguous MC-CDMA (NC-MC-CDMA). Rajbanshi et al. [47] investigated the performance of NC-OFDM and NC-MC-CDMA techniques for CR transceivers and made the following conclusions. With respect to PAPR, NC-OFDM exhibits the same problem as the conventional OFDM, while in MC-CDMA PAPR is reduced through frequency domain spreading. With respect to the nulling of subcarriers, NC-OFDM exhibits better error robustness compared to NC-MC-CDMA. In [48], space time block coding and modified interference cancellation techniques are considered for achieving antenna diversity and suppressing interference due to subcarrier deactivation in MC-CDMA based CR systems.

Attar et al. [49] proposed a cognitive MC-CDMA scheme which can adaptively change its transmission parameters, like bandwidth and power of sub-bands, according to the interference level in each sub-band. Compared to fixed-bandwidth sub-band deactivation method, the proposed method increases the spectral efficiency by excluding only the portion of the sub-band affected by the interference. In [50], the authors showed that, MC-CDMA is more suitable for underlay cognitive radio transmission. In MC-CDMA based underlay Cognitive Radio systems, the secondary user can spread its signal across the entire band, including that occupied by the primary user. The secondary user is allowed to use the spectrum of the primary user only when the interference received from the secondary user is less than the interference level that is tolerable by the primary receiver. Distributed sensing is not required in such wide-band multicarrier systems and hence, the DSA network can operate without a base station infrastructure and/or a signaling channel. The BER performance of MC-CDMA system over Rayleigh fading channel is shown in Figure 6 with spreading factor as 
$\mathrm{SF}=32$. As shown in Figure 6, when the number of subcarriers deactivated $\left(U_{d}\right)$ to avoid causing interference to primary users increases, the BER increases due to interference among different users. Thus, interference cancellation techniques are used in MC-CDMA systems [48] to suppress the interference caused due to subcarrier deactivation.

Figure 6. The BER performance of MC-CDMA system for varying number of deactivated subcarriers $(\mathrm{Ud})$.

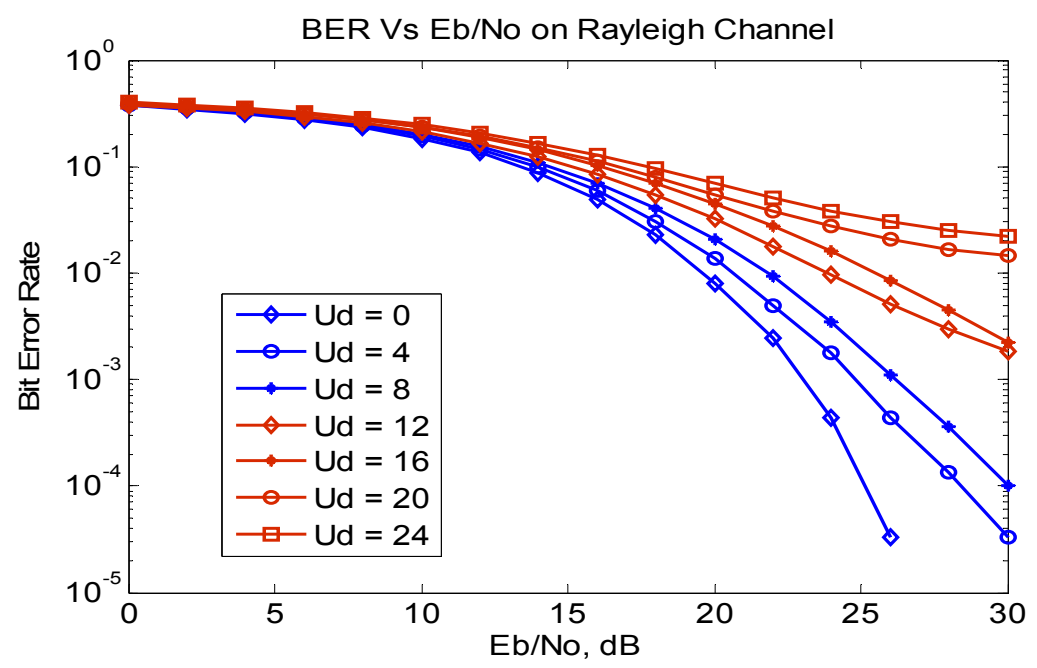

\subsection{SS-MC-CDMA}

Sarath et al. [51] proposed the Selective Subcarrier MC CDMA (SS-MC-CDMA) scheme which identifies the spectral holes and selects subcarriers in order to utilize them without interfering with the transmission of the primary user. In NC-MC-CDMA systems, adaptive subcarrier deactivation degrades the BER performance of the system, because the data in subcarriers are not independent as a result of the spreading operation. In the SS-MC-CDMA system, the data allocation to bins takes place only after the subcarriers are selected based on the channel state information and spectrum occupancy.

\section{3. $C I / M C-C D M A$}

In [52-54], the use of MC-CDMA with novel carrier interferometry (CI) complex spreading codes was proposed. The carrier interferometry MC-CDMA (CI/MC-CDMA) method, applied to cognitive radio systems, offers enhanced performance and flexibility compared to conventional MC-CDMA with WH spreading codes. In conventional MC CDMA systems with $N$ Walsh-Hadamard spreading codes, $N$ orthogonal users can be supported. If more than $N$ users are to be supported, pseudo-orthogonal codes must be employed. This results in high multiuser interference and degradation in bit-error rate (BER) performance due to cross correlation between pseudo-orthogonal codes. CI codes were introduced to overcome the above limitations of MC-CDMA. The advantages of CI codes include the availability of CI codes for any integer length and flexibility in adding additional number of users without much degradation in BER performance, which make CI/MC-CDMA more suitable for dynamic spectrum access environment. 


\section{4. $M C-D S-C D M A$}

Multicarrier direct sequence CDMA (MC-DS-CDMA) transmitter spreads the serial to parallel converted data streams in time domain using a spreading code [46], as shown in Figure 7. Thus, MC-DS-CDMA exploits time diversity in wireless channel to improve the BER performance of the system. Chang [55] proposed an interference avoidance code assignment strategy for the hierarchical 2-D spread MC-DS-CDMA based cognitive femtocell system. It was shown that, the proposed interference management scheme associated with the hierarchical system architecture can be a potential prototype of the CR-based femtocell system.

Figure 7. Reconfigurable MC-DS-CDMA transmitter.



Chang et al. [56] proposed an interweave cognitive radio (CR) system based on a hierarchical wide-band two-dimensional spread MC-DS-CDMA scheme. The authors proposed a DSA algorithm to utilize the white and gray spreading codes to improve the spectrum efficiency without harming the primary user transmission. Multi-user detection (MUD) techniques are necessary at the receiver side to combat the channel effects which lead to loss of orthogonality among different spreading codes and interference among different users' signals. In [57], MUD schemes based on the zero forcing (ZF), minimum mean square error (MMSE) and interference cancellation (IC) techniques were proposed for MC-DS-CDMA based CR systems. The advantages of these schemes include low-complexity, high-efficiency, high-flexibility, and robustness to imperfect channel knowledge. Diversity techniques are generally used to improve the BER performance of the MC-DS-CDMA system. Figure 8 shows the BER performance of the MC-DS-CDMA system over Rayleigh fading channel with transmit antenna diversity $(T)$ and frequency diversity $(S)$. We observe that the BER performance is significantly improved by increasing the number of transmit antennas $T$ from 1 to 2 . To improve the BER performance of the system especially for high order modulations, the frequency diversity order is increased by increasing $S$. However, when $T=2$ and $S$ becomes large, the improvement rate starts dropping, which is because the system approaches to its maximum capacity. Thus, considering trade-off with the complexity of the system due to the increased $S$, Figure 8 suggests that $S$ need not be larger than 4. Table 1 shows the comparison of various MCM schemes for CR systems. 
Table 1. Summary of the MCM Schemes for CR Systems: Their Advantages and Limitations.

\begin{tabular}{|c|c|c|}
\hline Technique & Advantages & Limitations \\
\hline $\begin{array}{l}\text { OFDM and } \\
\text { its variants }\end{array}$ & $\begin{array}{l}\text { 1. Easy implementation using FFT \& IFFT. } \\
\text { 2. Reconfigurability of parameters. } \\
\text { 3. Cyclic prefix allows simple one-tap channel equalization. } \\
\text { 4. Can be extended to a multiple access scheme } \\
\text { called OFDMA. } \\
\text { 5. Non contiguous blocks of subcarriers can be used for } \\
\text { transmission in NC-OFDM. } \\
\text { 6. NC-OFDM supports subcarrier deactivation without any } \\
\text { degradation in its error robustness. } \\
\text { 7. Interoperability with current wireless standards. }\end{array}$ & $\begin{array}{l}\text { 1. Spectral leakage due to large side lobes. } \\
\text { 2. Large PAPR except in Spread OFDM systems where PAPR is } \\
\text { reduced at the cost of increased computational complexity. } \\
\text { 3. Sensitive to frequency offsets. } \\
\text { 4. Loss in spectral efficiency due to cyclic prefix and guard bands. } \\
\text { 5. FFT does not provide sufficient spectral dynamic range for } \\
\text { spectrum sensing and hence may not be used for detecting low } \\
\text { power users. } \\
\text { 6. Hard to synchronize when the subcarriers are shared among } \\
\text { primary and secondary users. }\end{array}$ \\
\hline $\begin{array}{l}\text { FBMC and } \\
\text { its variants }\end{array}$ & $\begin{array}{l}\text { 1. No spectral leakage problem and hence there is no } \\
\text { adjacent carrier interference. } \\
\text { 2. Better spectral efficiency because of the elimination of } \\
\text { guard bands and cyclic prefix. } \\
\text { 3. More immune to narrowband interference. } \\
\text { 4. Filter bank-based spectral analyzer can provide high } \\
\text { spectral dynamic range for spectrum sensing. }\end{array}$ & $\begin{array}{l}\text { 1. Need for improved filter design. } \\
\text { 2. Increased latency. } \\
\text { 3. Increased computational complexity (equalizer and filters). } \\
\text { 4. MIMO-FBMC implementation is non-trivial compared to } \\
\text { MIMO-OFDM implementation. }\end{array}$ \\
\hline $\begin{array}{l}\text { MC-CDMA } \\
\text { and its } \\
\text { variants }\end{array}$ & $\begin{array}{l}\text { 1. Reduced PAPR compared to OFDM. } \\
\text { 2. Increased number of users supported through pseudo- } \\
\text { orthogonal codes. } \\
\text { 3. Improved BER performance using diversity gain in a } \\
\text { wireless channel. } \\
\text { 4. Suitable for underlay cognitive radio systems where } \\
\text { distributed spectrum sensing is not required. } \\
\text { 5. It can support variable data rate cognitive radio users } \\
\text { through variable spreading factor. }\end{array}$ & $\begin{array}{l}\text { 1. Subcarrier deactivation causes loss of orthogonality among } \\
\text { Walsh-Hadamard spreading codes and hence it causes degradation } \\
\text { in its error robustness. } \\
\text { 2. Requires synchronization in both time and frequency domains. } \\
\text { 3. Overcrowding of the spectrum due to spreading of each bit } \\
\text { using a spreading code. } \\
\text { 4. Increased complexity compared to OFDM. } \\
\text { 5. Requires multi user detection techniques in the uplink } \\
\text { transmission, because the code orthogonality among users will be } \\
\text { distorted in a frequency selective fading channel. }\end{array}$ \\
\hline
\end{tabular}

Figure 8. Bit error rate (BER) performance of the MC-DS-CDMA system.






\section{Efficient FFT Algorithms}

As discussed in the Sections 2.1 and 3.1, non-contiguous OFDM and non-contiguous MC-CDMA schemes are used in a DSA network to utilize non-contiguous blocks of subcarriers for the opportunistic transmission of secondary users. The CR transceivers deactivate some of the subcarriers in order to avoid interference with primary user's signal. Subcarriers are deactivated by assigning zero values to these subcarriers. Active subcarriers are assigned with data symbols. Thus, there are zero valued inputs for the IFFT and zero valued outputs for the FFT of the transceiver. When the number of zero valued inputs/outputs is more than the number of non-zero inputs/outputs, the conventional FFT/IFFT algorithms will not be efficient because of the unwanted operations on zero values. Therefore, the cognitive radio transceivers need some efficient FFT/IFFT algorithms that dynamically eliminate the multiplication and addition operations associated with deactivated subcarriers. Also, there are various efficient FFT algorithms that combine spreading and FFT operations. This section elaborates the efficient FFT algorithms proposed in the literature for cognitive radio systems.

\subsection{FFT Pruning}

The FFT pruning method was first proposed by Markel [58] for eliminating operations on zero input and output values which are not required. Later, several improved FFT pruning algorithms were proposed [59-63]. Sreenivas et al. [60] proposed an FFT algorithm for both input and output pruning. Since the computational structures of FFT and IFFT are same, most of these algorithms deal with FFT pruning. In all the pruning algorithms, the basic concept is to determine the index of the butterflies to be chosen for computations and those to be discarded. Thus, FFT computation time can be significantly reduced by pruning the operations on zero values in the discarded butterflies. Most of these algorithms are suitable for applications with specific zero-input or zero-output pattern distributions.

He et al. [63] applied the concept of FFT pruning to a multichannel OFDM system in order to achieve significant reduction of complexity. FFT pruning algorithm with frequency shift was proposed for the evaluation of comb spectrum in OFDM systems. As the comb spectrum shows considerable regularity, the proposed algorithm exploits the regularity for high efficiency. Also, the implementation of the proposed algorithm in a dedicated processor takes the advantage of the modularity of the algorithm. The assumption of zero values with a considerable regularity is not true for cognitive radio systems where subcarriers are switched off at random positions, based on the spectrum occupancy information and the sub channel condition. Alves et al. [64] proposed a general FFT pruning algorithm that operates on any zero-valued input distribution. An index matrix $M_{i}$ is generated based on the input values to the FFT. For a radix-2 FFT algorithm with $N$ levels ( $2^{N}$ FFT points), the matrix $M_{i}$ has $N$ columns and $2^{N}$ rows. Each element of the matrix corresponds to an addition/multiplication node of the FFT flow graph. If an element in the matrix is non-zero, the corresponding node has to be computed and if the element is zero, the corresponding node need not be computed. Thus, using the matrix the number of computations can be reduced. The reduction in computational complexity depends on the distribution of the non-zero inputs/outputs. 


\subsection{Modified FFT Pruning}

The FFT pruning algorithm proposed in [64] uses conditional statements. The time taken to execute these conditional statements often exceeds the time saved in reduction of computations. Thus, the algorithm does not yield an efficient FFT implementation in terms of computational time. Rajbanshi et al. [65] proposed a re-indexing algorithm and modified FFT pruning algorithm to avoid using the conditional statements and to reduce the computation time.

The modified FFT pruning algorithm is based on Cooley-Tukey divide-and-conquer algorithm [66]. The proposed modified pruning algorithm adds the pruning of unnecessary multiplication and addition operations, to the Cooley-Tukey algorithm in order to reduce the execution time. In this algorithm, first the matrix $M_{i}$ similar to the one in [64] is generated and based on which another matrix $M_{\text {index }}$ is constructed. The matrix $M_{\text {index }}$ consists of indices and the total number of nonzero elements in each column of matrix $M_{i}$. The proposed algorithm uses the information provided by $M_{\text {index }}$ to prune unnecessary computations. Using the simulation results it was shown that, the modified FFT pruning algorithm results in significant reduction in the mean execution time for calculating the FFT as compared to the conventional Cooley-Tukey algorithm for the sparseness factor of $60 \%$ or higher.

\subsection{Partial FFT Pruning}

Airoldi et al. [67] proposed an energy-efficient partial FFT pruning algorithm that allows for pruning of FFT inputs for cognitive radio systems. The proposed algorithm is based on modified FFT pruning algorithm [65], with reduction in the size of configuration matrix $M_{\text {index }}$ in order to lower the memory requirement for its storage. In the proposed algorithm, the size of the configuration matrix is reduced by a factor of 2 by storing only the identifier of the butterfly operations that must be performed, instead of storing the index of every non-zero input. Moreover, for a radix-2 $\mathrm{N}$-point FFT computation, by limiting the pruning operation to the first $\log _{2}(N)-3$ stages, the configuration matrix size can be further reduced. Thus, the proposed partial pruning FFT algorithm offers an optimal trade-off between performance in terms of computation time and memory requirement. In a dynamic spectrum access scenario, whenever there is a change in the indices of active subcarriers, the configuration matrix is re-evaluated and FFT computation is restarted. Figure 9 shows an example of 8-point decimation in frequency (DIF) FFT butterfly structure with complete and partial pruning. Considering a subset of $L$ non-zero input values, it was shown in [67] that the number of total multiplications and additions for executing a pruned $N$ point FFT can be reduced to

$$
\begin{aligned}
& M U L_{P R}=2 \times N \times \log _{2}(L)+2 \times N-4 \times L+\frac{(2 \times N \times L)}{2^{\left(\log _{2}(L)\right)}} \\
& A D D_{P R}=3 \times N \times \log _{2}(L)+3 \times N-6 \times L+\frac{(3 \times N \times L)}{2^{\left(\log _{2}(L)\right)}}
\end{aligned}
$$

If $L$ is contiguous and is power of two, then the number of multiplications and additions can be further reduced to

$$
\begin{aligned}
& M U L_{P R}=2 \times N \times \log _{2}(L) \\
& A D D_{P R}=3 \times N \times \log _{2}(L)
\end{aligned}
$$


Figure 9. An example FFT butterfly structure with pruning.



\subsection{Traced FFT Pruning}

Hu et al. [68] proposed a generic FFT pruning technique for the applications where the required outputs are very sparse. For example, in spectrum sensing one might be interested in sensing the spectrum corresponding to certain bins of the FFT or in narrow frequency bands. The proposed traced FFT pruning (TFFTP) technique does not require the outputs to be in continuous windows and thus can be applied for such spectrum sensing applications. A simple and effective method has been proposed to trace the computation path for the required outputs. For an $N$-point FFT computation there are $s=\log _{2} N$ stages in the signal flow graph. Initially the output nodes of the last stage are labeled from 0 to $N-1$. The output nodes of $(s-1)^{\text {th }}$ stage are divided into two groups and the nodes in each group are labeled from 0 to $N / 2-1$. The procedure is repeated until the first stage, where the nodes are divided into $N / 2$ groups and the output nodes of each group are labeled from 0 to 1 . Using the labels of the desired output nodes of the last stage, a bin set $\psi_{s}$ is formed. Other sets are derived iteratively as: $\psi_{s-1}=\psi_{s} \%(N / 2), \psi_{s-2}=\psi_{s-1} \%(N / 4)$, and so on until $\psi_{1}$ is obtained, where $\%$ is modulo operation. The sets $\psi_{1}, \psi_{2}, \ldots, \psi_{s}$ are the desired output node sets for the butterflies of stage 1 through stage $s$ respectively.

All the nodes on the signal flow graph are first labeled and the flow lines leading to the desired output nodes are joined to yield the computational path. Only the butterflies that lead to the desired output nodes in each stage are executed. The TFFTP technique can be applied to input pruning and also to simultaneous input and output pruning.

\subsection{Transform Decomposition}

The index used in FFT pruning algorithms shows irregularity because of the irregular position of zero inputs/outputs. This irregularity makes the hardware implementation of FFT pruning difficult. Sorensen et al. proposed an efficient algorithm called transform decomposition (TD) in [69]. It was shown that, transform decomposition is more efficient and flexible than FFT pruning in the view point of hardware implementation. Transform decomposition is considered as a modified Cooley-Tukey FFT, where the DFT is decomposed into two smaller DFTs. 
In the TD method, the $N$-point DFT with $L$ nonzero outputs is decomposed into two parts: $N_{2}$ number of $N_{1}$-point DFTs and the multiplications with twiddle factors and recombination of the products. Since only $L$ output values are nonzero, only $L$ twiddle factors are multiplied with each of the $N_{1}$-point DFTs. The total number of multiplications for TD method is given by

$$
M U L_{T D}=\frac{N}{2} \times \log _{2}\left(N_{1}\right)+L \times\left(N_{2}-1\right)
$$

Thus, there is a reduction of number of multiplications and the computational complexity. In a similar way, the transform decomposition algorithm can be applied for zero inputs. The advantage of transform decomposition is that, it does not need conditional statements to choose the butterfly for calculations as opposed to FFT pruning. Transform decomposition method exhibits considerable regularity which facilitates its hardware implementation.

Zhang et al. [70] proposed a computational structure of the transform decomposition algorithm for cognitive radio systems. In this method, efficient computation of the power-of-two FFT is considered for OFDM systems with only a subset of subcarriers being active. If there are $L$ active subcarriers, then there will be $L$ non-zero inputs/outputs for the IFFT/FFT blocks. Considering $N$ point FFT computation, $N_{1}$ is chosen to be the nearest power-of-two integer larger than $L$ and as a factor of $N$. As in [69], the computation is divided into two stages where, in the first stage $N_{1}$ point FFTs are computed and in the second stage multiplication with twiddle factors and recombination are performed. The computational structure shows a considerable regularity in memory addressing. Using simulation results it was shown that, the algorithm is more efficient for small number of non-zero output values $(L)$ and the complexity increases with the number of non-zero values.

Zhang et al. [71] proposed an efficient multi-resolution spectrum sensing method based on transform decomposition. The TD algorithm can be used to perform spectral analysis with finer resolutions in the frequency bands of interest, at low computational cost. Cui et al. [72] proposed a computationally efficient method for sparse input points DFT based on TD method and the method was shown to be more efficient than the conventional TD.

In [73], the authors have proposed a transform decomposition scheme based on two dimensional (2-D) systolic arrays for IDFT/DFT and IDCT/DCT, in order to achieve an efficient computation for OFDM in Cognitive Radio system. The authors have also developed a fast hybrid DFT and DCT architecture for OFDM systems.

\subsection{Split-radix FFT Pruning}

$\mathrm{Xu}$ et al. [74] proposed Split-radix FFT (SRFFT) pruning algorithm to reduce the computational complexity in the OFDM based cognitive radio, where zero valued inputs/outputs outnumber the non-zero inputs/outputs. The SRFFT pruning algorithm was shown to be more efficient than other pruning algorithms and Transform decomposition method in computational complexity. In this method, pruning is applied to split-radix FFT algorithm proposed in [75] instead of normal radix 2 FFT.

In split-radix FFT algorithm, an N-point DFT is decomposed into one N/2-point DFT and two N/4-point DFTs with twiddle factors. The decomposition is repeated for half- and quarter-length DFTs, until scalars result. The signal flow graph for SRFFT looks similar to that of radix-2 FFT except for the location of the twiddle factors. SRFFT offers reduction in complex multiplications compared to normal 
radix-2 FFT. Pruning the SRFFT corresponds to eliminating the computations that produce zero outputs. The general pruning proposed in [64], was applied in pruning the SRFFT with arbitrary length- $L$ nonzero outputs.

For an $N$-point DFT computation with $N=2^{r}$, a matrix of size $(N \times r)$ is generated with each element of the matrix corresponding to a node of the SRFFT flow graph. The elements determine whether a node value has to be computed or not. When the number of non-zero output is $L$ and is arbitrarily distributed, then total number of complex multiplications using SRFFT pruning is given by,

$$
M U L_{S R F F T}=\frac{L}{N} \times\left(\frac{N}{3} \times \log _{2}(N)-\frac{8}{9} \times(N-1)\right)
$$

Whereas, the number of multiplications for $N$-point radix-2 FFT and Split Radix FFT are given by

$$
\begin{gathered}
M U L_{\text {Radix }-2}=\frac{N}{2} \times \log _{2}(N) \\
M U L_{S R}=\frac{N}{3} \times \log _{2}(N)-\frac{8}{9} \times(N-1)
\end{gathered}
$$

\subsection{Fast Walsh-Hadamard-Fourier Transform}

Hamood et al. [76] proposed an efficient low computational complexity algorithm called Fast Walsh-Hadamard-Fourier Transform (FWFT) that combines the calculation of the Walsh-Hadamard transform (WHT) and the discrete Fourier transform (DFT). The FWFT algorithm can be used in Walsh-Hadamard spread orthogonal frequency division multiplexing systems (WHT-SOFDM) in order to increase the computational speed and to reduce the implementation cost. In conventional WHT-SOFDM systems the WHT and DFT are computed successively. However, the method is not efficient, because it requires the calculation of two transforms separately.

In the proposed method, both the transforms are combined into a single transform using a unified butterfly, so that the number of arithmetic operations, delays and indexing are reduced. The proposed algorithm is based on the sparse matrices factorization method using the Kronecker product technique. The radix-4 FWFT algorithm is used for the fast calculation of a WHT-DFT sequence whose length equals to a power of four. By comparing the simulation results of the proposed algorithm with conventional radix-4 WHT-FFT algorithm, it was shown that radix-4 FWFT achieves a significant reduction in the number of arithmetic operations and computer run time.

\subsection{T-Transform}

Ahmed et al. [77] proposed a multicarrier system using low computational complexity transform called T-Transform that combines WHT and DFT into a single fast orthonormal unitary transform. The proposed algorithm offers a significant improvement in BER and reasonable reduction in the PAPR. Through the proposed algorithm, OFDM systems can achieve high frequency diversity gain by combining all the data samples resulting in the transmission over many subcarriers. The frequency diversity of the proposed T-OFDM system results in increased resilience over multipath fading channels. The proposed transform reduces PAPR by reducing the high peak power, while preserving the average transmitted power and data rate. 
T-transform is based on the factorization of the DFT and WHT matrices using the Kronecker product technique. Advantages of the T-transform include simple structure and low computational complexity. For example in radix-2, 16-point FFT butterfly diagram the number of stages will be four. However, the proposed T-transform butterfly diagram has only three stages and in each stage many of the butterflies are zero and do not need to be calculated. Unlike FWFT, the T-transform can be scaled up or down to any transform length.

\subsection{Sparse FFT}

Schneider [78] proposed a new FFT algorithm called sparse FFT that is applicable for sparse signals which contain only a relatively small number of frequency components of significant size. In spectral analysis most of the Fourier coefficients of a signal are small or equal to zero, i.e., the output of the DFT is sparse. When the output of the DFT is sparse or approximately sparse, it is desired to have an output-sensitive algorithm, whose runtime depends on the number of significant coefficients computed. The disadvantage with conventional FFT is that it takes the same amount of computational time for any input signal. The proposed sparse FFT algorithm can reduce much of the computational complexity for sparse signals compared to conventional FFT, especially when the number of frequency components is a single-digit percentage of the number of samples.

In cognitive radio systems spectrum sensing is done for a wide bandwidth typically in GHz. Sampling becomes expensive and analysis require heavy computations, when the bandwidth is large. Spectral compressive sensing techniques based on the proposed sparse FFT [79] can achieve low cost and high speed sensing of spectrum by leveraging the sparsity of the spectrum. The proposed algorithm is not iterative unlike other typical approaches in which large coefficients are first estimated and are subtracted from ori the $k$ largest coefficients simultaneously. In addition, ginal signal and the remainder are processed recursively. The proposed algorithm identifies and estimates whether the algorithm does not need interpolation of sample points. Thus, the algorithm has been proven to be efficient by avoiding both iteration and interpolation. The algorithm is structurally simpler than other algorithms and as a consequence the algorithm is faster in computing FFT for sparse signals.

For an input signal of length $N$, which has $K$ non-zero entries in the Fourier transform of the signal, sparse FFT costs $O(K \log N)$ instead of $O(N \log N)$ in FFT. In [80], a fast sparse FFT algorithm has been proposed, that costs $O(K \log K)$ averagely. In the proposed method, the original input signal is downsampled at the beginning and the Fourier transform of the downsampled signal is used to locate and estimate $K$ non-zero entries of the Fourier transform of the original signal.

\section{Summary of Efficient FFT Algorithms}

The pruning algorithms proposed in [58-63], work for specific zero-input/output distributions. A more generic algorithm that works for any zero valued input distribution was proposed in [64]. The conditional statements were used for deciding whether a node in the signal flow graph has to be computed or not. The time taken to execute the conditional statements reduces the efficiency of the FFT. Thus, modified pruning algorithm was proposed to avoid using conditional statements [65]. The configuration matrix used in [65] requires high data storage capabilities for large size FFTs. The configuration matrix size used for defining the nodes to be executed is reduced by introducing butterfly 
identifier and using partial FFT pruning in [67]. Thus, there was a trade-off between complexity reduction and memory requirement.

Split Radix FFT pruning algorithm [74] is able to reduce the computational complexity by applying pruning to split radix FFT instead of radix-2 FFT. It was shown in [74] that, the SRFFT pruning technique has the least computational complexity for any arbitrary distributions of the zero inputs/outputs. However, all these FFT pruning algorithms require some preprocessing operational overhead. In Traced FFT Pruning algorithm [68], the preprocessing operation is made simple and recursive. In the application of OFDM based cognitive radio systems, the hardware implementation of FFT pruning algorithms become complex due to arbitrary distributions of the zero inputs/outputs. Hence, Transform decomposition method was proposed in $[69,70]$, which is more efficient and flexible than FFT pruning algorithms in the view point of hardware implementation.

Algorithms proposed in [76,77], are used for WHT-spread OFDM systems. These algorithms combine WHT and DFT computations into a single transform. While FWFT is applicable for computing transforms of length in power of four, T-transform can be applied for the computation of transforms of any length. Sparse FFT algorithm proposed in [78-80] is suitable for any signal that is sparse in frequency domain and hence, the algorithm is much suitable for compressive spectrum sensing.

\section{Conclusions}

In the first part of this contribution, advantages and limitations of the variants of spread spectrum schemes and multicarrier modulation techniques are discussed. Based on the type of cognitive radio system, the modulation scheme is selected. Generally, for spectrum interweave cognitive radio systems, variants of MCM schemes are preferred and for spectrum underlay cognitive radio systems, variants of SS techniques are preferred. MCSSM schemes are used in both interweave and underlay CR systems. The second part of this contribution gives an elaborate survey of various modified FFT algorithms proposed in the literature for cognitive radio applications. These algorithms offer efficiency in terms of energy consumption and time taken for execution when there are zero valued inputs/outputs for the IFFT/FFT of the modem. The pruning algorithms were able to achieve much reduction in computational complexity but in the view point of hardware implementation transform decomposition technique is more efficient and flexible. The modified FFT algorithms require efficient hardware implementation methods. Hence, in recent years much research has been carried out in designing parallel, pipelined and reconfigurable FFT architectures for multi-standard cognitive radio systems.

\section{Author Contributions}

Both Mohandass Sundararajan and Umamaheswari Govindaswamy contributed to the literature survey, MATLAB simulations, interpretation of results and manuscript preparation. Both authors read and approved the final manuscript.

\section{Conflicts of Interest}

The authors declare no conflict of interest. 


\section{References}

1. Mitola, J., III. Cognitive radio for flexible mobile multimedia communications. In Proceedings of the International Workshop on Mobile Multimedia Communications, San Diego, CA, USA, 15-17 November 1999; pp. 3-10.

2. Haykin, S. Cognitive Radio: Brain-empowered Wireless Communications. IEEE J. Sel. Areas Commun. 2005, 23, 201-220.

3. Benmammar, B.; Amraoui, A.; Krief, F. A Survey on Dynamic Spectrum Access Techniques in Cognitive Radio Networks. Int. J. Commun. Netw. Inf. Security 2013, 5, 68-79.

4. Hu, S.; Yao, Y.-D.; Yang, Z. MAC protocol identification using support vector machines for cognitive radio networks. IEEE Wireless Commun. 2014, 21, 52-60.

5. Yucek, T.; Arslan, H. A Survey of Spectrum Sensing Algorithms for Cognitive Radio Applications. IEEE Commun. Surveys Tuts. 2009, 11, 116-130.

6. Farhang-Boroujeny, B.; Kempter, R. Multicarrier Communication Techniques for Spectrum Sensing and Communication in Cognitive Radios. IEEE Commun. Mag. 2008, 46, 80-85.

7. Akyildiz, I.F.; Lee, W.Y.; Vuran, M.C.; Mohanty, S. Next Generation Dynamic Spectrum Access Cognitive Radio Wireless Networks: A Survey. Elsevier J. Comput. Netw. 2006, 50, 2127-2159.

8. Luo, T.; Jiang, T.; Xiang, W.; Chen, H.H. A Subcarriers Allocation Scheme for Cognitive Radio Systems Based on Multi-carrier Modulation. IEEE Trans. Wireless Commun. 2008, 7, 3335-3340.

9. Weiss, T.A.; Jondral, F.K. Spectrum pooling: An innovative strategy for the enhancement of spectrum efficiency. IEEE Commun. Mag. 2004, 42, S8-S14.

10. Mahmoud, H.A.; Yucek, T.; Arslan, H. OFDM for Cognitive Radio: Merits and Challenges. IEEE Trans. Wireless Commun. 2009, 16, 6-15.

11. Cheng, P.; Zhang, Z.Y.; Huang, H.; Qiu, P. A distributed algorithm for optimal resource allocation in cognitive OFDMA systems. In Proceedings of IEEE International Conference on Communications, Beijing, China, 19-23 May 2008; pp. 4718-4723.

12. Zhang, Y.; Leung, C. Resource Allocation in an OFDM-Based Cognitive Radio System. IEEE Trans. Commun. 2009, 57, 1928-1931.

13. Xu, X.; Yao, Y.-D.; Hu, S.; Yao, Y. Joint subcarrier and bit allocation for secondary user with primary users' cooperation. KSII Trans. Int. Inf. Syst. 2013, 7, 3037-3054.

14. Pagadarai, S.; Wyglinski, A.M.; Rajbanshi, R. A Novel Sidelobe Suppression Technique for OFDM-Based Cognitive Radio Transmission. In Proceedings of IEEE Symposium on New Frontiers in Dynamic Spectrum Access Networks, Chicago, IL, USA, 14-17 October 2008; pp. 1-7.

15. Fu, X.; Wang, J.; Li, S.Q. Sidelobe suppression for OFDM based cognitive radio systems. In Proceedings of the International Conference on Communications and Networking in China, Xian, China, 26-28 August 2009; pp. 1-5.

16. Brandes, S.; Cosovic, I.; Schnell, M. Reduction of out-of-band radiation in OFDM systems by insertion of cancellation carriers. IEEE Commun. Lett. 2006, 10, 420-422.

17. Mahmoud, H.A.; Arslan, H. Sidelobe suppression in OFDM based spectrum sharing systems using adaptive symbol transition. IEEE Commun. Lett. 2008, 12, 133-135. 
18. Ghassemi, A.; Gulliver, T.A. PAPR reduction in OFDM based cognitive radio with blockwise-subcarrier activation. In Proceedings of IEEE International Conference on Communications, Ottawa, ON, Canada, 10-15 June 2012; pp. 5598-5602.

19. Rajbanshi, R.; Wyglinski, A.M.; Minden, G.J. Adaptive-Mode Peak-to-Average Power Ratio Reduction Algorithm for OFDM-Based Cognitive Radio. In Proceedings of IEEE Vehicular Technology Conference, Montreal, Canada, 25-28 September 2006; pp. 1-5.

20. Poston, J.D.; Horne, W.D. Discontiguous OFDM considerations for dynamic spectrum access in idle TV channels. In Proceedings of IEEE International Symposium on New Frontiers in Dynamic Spectrum Access Networks, Baltimore, MD, USA, 8-11 November 2005; pp. 607-610.

21. Wylie-Green, M.P. Dynamic spectrum sensing by multiband OFDM radio for interference mitigation. In Proceedings of IEEE International Symposium on New Frontiers in Dynamic Spectrum Access Networks, Baltimore, MD, USA, 8-11 November 2005; pp. 619-625.

22. Tang, H. Some physical layer issues of wide-band cognitive radio systems. In Proceedings of IEEE International Symposium on New Frontiers in Dynamic Spectrum Access Networks, Baltimore, MD, USA, 8-11 November 2005; pp. 151-159.

23. Park, M.; Jun, H.; Cho, J.; Cho, N.; Hong, D.; Kang, C. PAPR reduction in OFDM transmission using Hadamard transform. In Proceedings of IEEE International Conference on Communications, New Orleans, LA, USA, 18-22 June 2000; pp. 430-433.

24. Dlugaszewski, Z.; Wesolowski, K. WHT/OFDM-An improved OFDM transmission method for selective fading channels. In Proceedings of IEEE Symposium on Communications and Vehicular Technology, Leuven, Belgium, 19 October 2000; pp. 144-149.

25. Wu, Y.; Ho, C.K.; Sun, S. On some properties of Walsh-Hadamard transformed OFDM. In Proceedings of IEEE Vehicular Technology Conference-Fall, Vancouver, BC, Canada, 24-28 September 2002; pp. 2096-2100.

26. Shaopeng, W.; Shihua, Z.; Guomei, Z. A Walsh-Hadamard coded spectral efficient full frequency diversity OFDM system. IEEE Trans. Commun. 2010, 58, 28-34.

27. Li, X.; Chakravarthy, V.D.; Wang, B.; Wu, Z. Spreading Code Design of Adaptive Non-Contiguous SOFDM for Dynamic Spectrum Access. IEEE J. Sel. Topics Signal Process. 2011, 5, 190-196.

28. Zhang, C. Non-continuous carrier-interferometry codes. In Proceedings of International Workshop on Signal Design and its Application in Communications, Fukuoka, Japan, 19-23 October 2009; pp. 134-137.

29. Wiegandt, D.A.; Wu, Z.-Q.; Nassar, C.R. High-throughput, high performance OFDM via pseudo-orthogonal carrier interferometry spreading codes. IEEE Trans. Commun. 2003, 51, 1123-1134.

30. Kollar, Z.; Horvath, P. Physical Layer Considerations for Cognitive Radio: Modulation Techniques. In Proceedings of IEEE Vehicular Technology Conference (VTC Spring), Yokohama, Japan, 15-18 May 2011; pp. 1-5.

31. Myung, H.G.; Lim, J.; Goodman, D.J. Single Carrier FDMA for Uplink Wireless Transmission. IEEE Veh. Technol. Mag. 2006, 1, 30-38.

32. Farhang-Boroujeny, B. Ofdm versus filter bank multicarrier. IEEE Signal Process. Mag. 2011, $28,92-112$. 
33. Elezabi, A.; Kashef, M.; Abdallah, M.; Khairy, M.M. Cognitive interference-minimizing code assignment for underlay CDMA networks in asynchronous multipath fading channels. In Proceedings of the 2009 International Conference on Wireless Communications and Mobile Computing (IWCMC '09), Leipzig, Germany, 21-24 June 2009; pp. 1279-1283.

34. Elezabi, A.; Kashef, M.; Abdallah, M.; Khairy, M. CDMA underlay network with cognitive interference-minimizing code assignment and semi-blind interference suppression. Wirel. Commun. Mob. Comput. 2009, 9, 1460-1471.

35. Wang, B.; Zhao, D. Performance analysis in CDMA-based cognitive wireless networks with spectrum underlay. In Proceedings of IEEE Global Telecommunication conference (GLOBECOM), New Orleans, LA, USA, 30 November-4 December 2008; pp. 1-6.

36. Hoang, A.T.; Liang, Y.-C. A two-phase channel and power allocation scheme for cognitive radio networks. In Proceedings of the IEEE 17th International conference on Personal, Indoor and Mobile Radio Communications, Helsinki, Finland, 11-14 September 2006; pp. 1-5.

37. Zhang, X.; Su, H. Opportunistic spectrum sharing schemes for CDMA-based uplink MAC in cognitive radio networks. IEEE J. Sel. Areas Commun. 2011, 29, 716-730.

38. Li, M.; Batalama, S.N.; Pados, D.A.; Melodia, T.; Medley, M.J.; Matyjas, J.D. Cognitive code-division links with blind primary-system identification. IEEE Trans. Wireless Commun. 2011, 10, 3743-3753.

39. Yang, B.; Feng, G.; Shen, Y.; Long, C.; Guan, X. Channel-aware access for cognitive radio networks. IEEE Trans. Veh. Technol. 2009, 58, 3726-3737.

40. Dashti, M.; Azmi, P. Joint power and rate allocation in CDMA-based underlay cognitive radio networks for a mixture of streaming and elastic traffic. EURASIP J. Wirel. Commun. Netw. 2012, 2012, 262-272.

41. Roy, S.D.; Upadhayay, N.K.; Kundu, S. Performance of Joint Admission and Power Control Algorithms for Cognitive Radio CDMA Networks in Shadowed Environment. Int. J. of Comm. Net. Sec. 2011, 1, 1-6.

42. Roy, S.D.; Kundu, S.; Ferrari, G.; Raheli, R. Cognitive radio CDMA networking with spectrum sensing. Int. J. Commun. Syst. 2012, doi:10.1002/dac.2421.

43. Roy, S.D.; Kundu, S.; Ferrari, G.; Raheli, R. On spectrum sensing in cognitive radio CDMA networks with beamforming. Elsevier Phys. Commun. 2013, 9, 73-87.

44. Hussein, N.M. Modified Greedy Algorithm for Cognitive DS-CDMA System. Int. J. Adv. Res. Comput. Commun. Eng. 2013, 2, 2321-2326.

45. Sarath, D.; Nolan, K.E.; Sutton, P.D.; Doyle, L.E. Exploring The Reconfigurability Options of Multi-Carrier CDMA in Cognitive Radio Systems. In Proceedings of IEEE International Symposium on Personal, Indoor and Mobile Radio Communications, Athens, Greece, 3-7 September 2007; pp. 1-5.

46. Prasad, R.; Hara, S. Overview of multicarrier CDMA. IEEE Commun. Mag. 1997, 35, 126-133.

47. Rajbanshi, R.; Chen, Q.; Wyglinski, A.M.; Minden, G.J.; Evans, J.B. Quantitative comparison of agile modulation techniques for cognitive radio transceivers. In Proceedings of IEEE Consumer Communications and Networking Conference, Las Vegas, NV, USA, 11-13 January 2007; pp. $1144-1148$. 
48. Mohandass, S.; Umamaheswari, G. Modelling and Simulation of Interference Cancellation Receiver for MIMO Multicarrier CDMA based Cognitive Radio. Int. Rev. Mod. Sim. 2014, 7, 196-205.

49. Attar, A.; Nakhai, M.R.; Aghvami, A.H. Cognitive Radio Transmission Based on Direct Sequence MC-CDMA. IEEE Trans. Wirel. Commun. 2008, 7, 1157-1162.

50. Luo, T.; Lin, F.; Jiang, T.; Guizani, M.; Chen, W. Multicarrier modulation and cooperative communication in multihop cognitive radio networks. IEEE Wireless Commun. 2011, 18, 38-45.

51. Sarath, D.; Nolan, K.E.; Sutton, P.D.; Doyle, L.E. Enabling Dynamic Spectrum Access using SS-MC-CDMA. In Proceedings of International Conference on Cognitive Radio Oriented Wireless Networks and Communications, Orlando, FL, USA, 1-3 August 2007; pp. 193-198.

52. Natarajan, B.; Nassar, C.R.; Shattil, S.; Michelini, M.; Wu, Z. High-Performance MC-CDMA via Carrier Interferometry Codes. IEEE Trans. Veh. Technol. 2001, 50, 1344-1353.

53. Natarajan, B.; Wu, Z.; Nassar, C.R.; Shattil, S. Large Set of CI Spreading Codes for High-Capacity MC-CDMA. IEEE Trans. Commun. 2004, 52, 1862-1866.

54. Mukherjee, M.; Kumar, P. Design and performance of WH-spread CI/MC-CDMA with iterative interference cancellation receiver. Elsevier Phys. Commun. 2012, 5, 217-229.

55. Chang, C.W. An Interference-Avoidance Code Assignment Strategy for the Hierarchical Two-Dimensional-Spread MC-DS-CDMA System: A Prototype of Cognitive Radio Femtocell System. IEEE Trans. Veh. Technol. 2012, 61, 166-184.

56. Chang, C.W.; Kuo, C.C. An Interweave Cognitive Radio System Based on the Hierarchical 2D-Spread MC-DS-CDMA. In Proceedings of IEEE Vehicular Technology Conference Fall, Ottawa, ON, Canada, 6-9 September 2010; pp. 1-5.

57. Yang, L.L.; Wang, L.C. Zero-forcing and minimum mean-square error multiuser detection in generalized multicarrier DS-CDMA systems for cognitive radio. EURASIP J. Wirel. Commun. Netw. 2008, 2008, 1-13.

58. Markel, J. FFT Pruning. IEEE Trans. Audio Electroacoust. 1971, 19, 305-311.

59. Skinner, D.P. Pruning the decimation-in-time FFT algorithm. IEEE Trans. Acoust. Speech Signal Process. 1976, 24, 193-194.

60. Sreenivas, T.V.; Rao, P.V.S. FFT algorithm for both input and output pruning. IEEE Trans. Acoust. Speech Signal Process. 1979, 27, 291-292.

61. Jaroslavski, L.P. Comments on FFT algorithm for both input and output pruning. IEEE Trans. Acoust. Speech Signal Process. 1981, 29, 448-449.

62. Holm, S. FFT pruning applied to time domain interpolation and peak localization. IEEE Trans. Acoust. Speech Signal Process. 1987, 35, 1776-1778.

63. He, S.; Torkelson, M. Computing Partial DFT for Comb Spectrum Evaluation. IEEE Signal Process. Lett. 1996, 3, 173-175.

64. Alves, R.G.; Osorio, P.L.; Swamy, M.N.S. General FFT Pruning Algorithm. In Proceedings of IEEE Midwest Symposium on Circuits and Systems, Lansing, MI, USA, 8-11 August 2000; pp. 1192-1195. 
65. Rajbanshi, R.; Wyglinski, A.M.; Minden, G.J. An Efficient Implementation of NC-OFDM Transceivers for Cognitive Radios. In Proceedings of International Conference on Cognitive Radio Oriented Wireless Networks and Communications, Mykonos Island, Greece, 8-10 June 2006; pp. 1-5.

66. Cooley, J.W.; Tukey, J.W. An algorithm for the machine calculation of complex Fourier series. Math. Comput. 1965, 19, 297-301.

67. Airoldi, R.; Anjum, O.; Garzia, F.; Nurmi, J.; Wyglinski, M. Energy-efficient Fast Fourier Transforms for Cognitive Radio Systems. IEEE Micro 2010, 30, 66-76.

68. Hu, Z.; Wan, H. A Novel Generic Fast Fourier Transform Pruning Technique and Complexity Analysis. IEEE Trans. Signal Process. 2005, 53, 274-282.

69. Sorensen, H.V.; Burrus, S. Efficient Computation of the DFT with Only a Subset of Input or Output Points. IEEE Trans. Signal Process. 1993, 41, 1184-1200.

70. Zhang, Q.; Kokkeler, A.B.J.; Smit, G.J.M. An Efficient FFT for OFDM Based Cognitive Radio on a Reconfigurable Architecture. In Proceedings of the IEEE International Conference on Communication, Glasgow, 24-28 June 2007; pp. 6522-6526.

71. Zhang, Q.; Kokkeler, A.B.J.; Smit, G.J.M. An efficient multi-resolution spectrum sensing method for cognitive radio. In Proceedings of the International Conference on Communications and Networking in China, Hangzhou, China, 25-27 August 2008; pp. 1226-1229.

72. Cui, Y.; Zhao, Z.; Zhang, H. An efficient transform decomposition method for sparse input points DFT in multiple-carriers cognitive radio system. In Proceedings of the International Symposium on Communications and Information Technologies, Tokyo, Japan, 26-29 October 2010; pp. 1200-1204.

73. Chen, Z.; Lee, M.H.; Kim, C.J. Fast Hybrid DFT/DCT Architecture for OFDM in Cognitive Radio System. In Proceedings of the International Conference on Future Generation Communication and Networking, Jeju Island, Korea, 6-8 December 2007; pp. 301-306.

74. Xu, Y.; Lim, M.S. Split-Radix FFT pruning for the reduction of computational complexity in OFDM based Cognitive Radio system. In Proceedings of the International Symposium on Communications and Information Technology, Icheon, Korea, 28-30 September 2009; pp. 421-424.

75. Duhamel, P.; Hollmann, H. Split-radix FFT algorithm. Electron. Lett. 1984, 20, 14-16.

76. Hamood, M.T.; Boussakta, S. Fast Walsh Hadamard Fourier Transform Algorithm. IEEE Trans. Signal Process. 2011, 59, 5627-5631.

77. Ahmed, M.S.; Boussakta, S.; Sharif, B.S.; Tsimenidis, C.C. OFDM Based on Low Complexity Transform to Increase Multipath Resilience and Reduce PAPR. IEEE Trans. Signal Process. 2011, 59, 5994-6007.

78. Schneider, D. A Faster Fast Fourier Transform: New algorithm crunches sparse data with speed. IEEE Spectr. 2012, 49, 12-13.

79. Hassanieh, H.; Indyk, P.; Katabi, D.; Price, E. Simple and Practical Algorithm for Sparse Fourier Transform. In Proceedings of the ACM-SIAM Symposium on Discrete Algorithms, Kyoto, Japan, 17-19 January 2012; pp. 1183-1194. 
80. Hsieh, S.H.; Lu, C.S.; Pei, S.C. Sparse Fast Fourier Transform by downsampling. In Proceedings of the IEEE International Conference on Acoustics, Speech and Signal Processing, Vancouver, BC, Canada, 26-31 May 2013; pp. 5637-5641.

(C) 2014 by the authors; licensee MDPI, Basel, Switzerland. This article is an open access article distributed under the terms and conditions of the Creative Commons Attribution license (http://creativecommons.org/licenses/by/3.0/). 